\title{
A Sampling Method for Determining the Lowest Eigenvalue and the Principal Eigenfunction of Schrödinger's Equation ${ }^{1}$
}

\author{
By M. D. Donsker and M. Kac
}

\begin{abstract}
This is a preliminary report on a sampling method for finding the lowest eigenvalue and the corresponding eigenfunction of Schrödinger's equation. The theory underlying the procedure is explained and two cases treated numerically. Although the initial results are encouraging, more experimentation will be needed to fully test the practicality of the method.
\end{abstract}

\section{Introduction}

Certain problems leading to complicated partial or integro-differential equations have recently been approached and some actually solved by utilizing various probability techniques and sampling methods. Collectively these methods have become known as the "Monte Carlo" method.

The problems to which Monte Carlo techniques have been applied seem to be divided into two types. Typical of the first type is the problem of neutrons diffusing in material media in which the particles are subjected not only to certain deterministic influences but to random influences as well. In such a problem, the Monte Carlo approach consists in permitting a "particle" to play a game of chance, the rules of the game being such that the actual deterministic and random features of the physical process are step by step exactly imitated by the game. By considering very large numbers of particles, one can answer such questions as the distribution of the particles at the end of a certain period of time, the number of particles to escape through a shield of specified thickness, etc. One important characteristic of the preceding approach is that the functional equation describing the diffusion process is bypassed completely, the probability model used being derived from the process itself.

A more sophisticated application of Monte

\footnotetext{
1 The preparation of this paper was sponsored (in part) by the Office of Naval Research.
}

Carlo methods is to the problem of finding a probability model or game whose solution is related to the solution of a partial differential equation, or, as in the present paper, to determine the least eigenvalue of a differential operator by means of a sampling process. As an example of how the latter problem might be attacked, we quote from a paper of Metropolis and Ulam: ${ }^{2}$

"For example, as suggested by Fermi, the time independent Schrödinger equation

$$
\Delta \phi(x, y, z)=(\lambda-V) \phi(x, y, z),
$$

could be studied as follows. Reintroduce time dependence by considering

$$
u(x, y, z, t)=\phi(x, y, z) e^{-\lambda t} ;
$$

then, $u$ will obey the equation

$$
\frac{\partial u}{\partial t}=\Delta u-V u
$$

This last equation can be interpreted, however, as describing the behavior of a system of particles each of which performs a random walk, i. e., diffuses isotropically and at the same time is subject to multiplication, which is determined by the value of the point function $V$. If the solution of the latter equation corresponds to a spatial

${ }^{2} \mathrm{~N}$. Metropolis and S. Ulam, The Monte Carlo method, J. Am. Stat. Assn. 44, 247, pp. 335 to 341 (Sept. 1949). 
mode multiplying exponentially in time, the examination of the spatial part will give the desired $\phi(x, y, z)$-corresponding to the lowest 'eigenvalue' $\lambda . "{ }^{3}$

The main purpose of the present paper is to present an alternative method for finding the lowest eigenvalue and corresponding eigenfunction of Schrödinger's equation. The chief difference between the two approaches is that ours involves only a random walk eliminating entirely the multiplicative process. This alteration in the model seems to simplify the numerical aspects of the problem, especially if punched card equipment is to be used. Apart from the possible numerical simplification, the method is based on a mathematical theory that in itself is of some interest.

\section{Mathematical Theory}

Let $X_{1}, X_{2}, X_{3}, \ldots$ be independent identically distributed random variables each having mean 0 and standard deviation 1 and let $S_{k}=X_{1}+X_{2}+$ ... $+X_{k}$. Under certain general assumptions on $V(x)$, the most severe of which is that $V(x)$ be nonnegative, it can be shown ${ }^{4}$ that the limiting distribution function $\sigma(\alpha, t)$ of the random variable

$$
\frac{1}{n} \sum_{k<n t} V\left(\frac{S_{k}}{\sqrt{n}}\right)
$$

is such that

$$
\int_{0}^{\infty} \int_{0}^{\infty} e^{-\alpha-s t} d_{\alpha} \sigma(\alpha, t) d t=\int_{-\infty}^{\infty} \psi(x) d x
$$

where $\psi(x)$ is the fundamental solution of the differential equation

$$
\frac{1}{2} \frac{d^{2} \psi}{d x^{2}}-(s+V(x)) \psi=0,
$$

subject to the conditions

$$
\begin{gathered}
\psi(x) \rightarrow 0 \quad x \rightarrow \pm \infty \\
\left|\psi^{\prime}(x)\right|<M \quad x \neq 0 \\
\psi^{\prime}(+0)-\psi^{\prime}(-0)=-2 .
\end{gathered}
$$

The fundamental solution $\psi(x)$ of (3) is expressible in terms of the normalized eigenfunction

\footnotetext{
${ }^{3}$ We have since learned that Dr. G. W. King has made similar calculalations using a closely related method.

4 M. Kac, On distributions of certain Wiener functionals, Trans. Am. Math. Soc. 65, 1 to 13 (1949).
}

$\left\{\left(\psi_{j}(x)\right\}\right.$ and eigenvalues $\lambda_{j}$ of the one-dimensional Schrödinger eigenvalue problem ${ }^{5}$

$$
\frac{1}{2} \frac{d^{2} \psi}{d x^{2}}-V(x) \psi(x)=-\lambda \psi \quad \lambda>0
$$

as

$$
\psi(x)=\sum_{j} \frac{\psi_{j}(0) \psi_{j}(x)}{s+\lambda_{j}} .
$$

Thus from (5 and 2)

$$
\begin{aligned}
\int_{-\infty}^{\infty} \psi(x) d x & =\int_{0}^{\infty} \int_{0}^{\infty} e^{-\alpha-s t} d_{\alpha} \sigma(\alpha, t) d t \\
& =\sum_{j} \frac{\psi_{j}(0) \int_{-\infty}^{\infty} \psi_{j}(x) d x}{s+\lambda_{j}} .
\end{aligned}
$$

Inverting (6) with respect to $s$ we get,

$$
\int_{0}^{\infty} e^{-\alpha} d_{\alpha} \sigma(\alpha, t)=\sum_{j} e^{-\lambda_{j} t} \psi_{\jmath}(0) \int_{-\infty}^{\infty} \psi_{j}(x) d x,
$$

and therefore we obtain the expression for $\lambda_{1}$,

$$
\lambda_{1}=\lim _{t \rightarrow \infty}-\frac{1}{t} \log \int_{0}^{\infty} e^{-\alpha} d_{\alpha} \sigma(\alpha, t) .
$$

If in (7) we neglect all terms in the expansion but the first, we obtain

$$
\log \int_{0}^{\infty} e^{-\alpha} d_{\alpha} \sigma(\alpha, t) \sim \log \left\{\psi_{1}(0) \int_{-\infty}^{\infty} \psi_{1}(x) d x\right\}-\lambda_{1} t
$$

or

$$
\lambda_{1} \sim \frac{\log \left\{\psi_{1}(0) \int_{-\infty}^{\infty} \psi_{1}(x) d x\right\}}{t}-\frac{1}{t} \log \int_{0}^{\infty} e^{-\alpha} d_{\alpha} \sigma(\alpha, t) .
$$

Thus, if, by choosing a finite $t$, we attempt to calculate $\lambda_{1}$ from (8), we have two sources of error. The first, usually a small source of error, is from the exponentials neglected in the expansion (7). The second, and more important, is from neglecting the term $(1 / i) \log \left\{\psi_{1}(0) \int_{-\infty}^{\infty} \psi_{1}(x) d x\right\}$. This latter source of error is especially significant, since, as will be apparent shortly, it is impractical from other points of view to take $t$ very large. All of this difficulty may be obviated by considering (7) for two distinct values of $t$, say $t_{1}$ and $t_{2}$, then, if

\footnotetext{
${ }_{3}^{3}$ From here on all the steps are formal. In all cases of physical interest they can be justified rigorously.
} 
we neglect the exponentials after the first as before, we get on dividing

$$
\lambda_{1} \sim \frac{1}{t_{2}-t_{1}} \log \frac{\int_{0}^{\infty} e^{-\alpha} d_{\alpha} \sigma\left(\alpha, t_{1}\right)}{\int_{0}^{\infty} e^{-\alpha} d_{\alpha} \sigma\left(\alpha, t_{2}\right)} .
$$

The Monte Carlo process consists in the calculation of $\sigma\left(\alpha, t_{1}\right)$ and $\sigma\left(\alpha, t_{2}\right)$ by a sampling process.

If instead of $\sigma(\alpha, t)$ we consider the limiting distribution $\sigma_{\xi}(\alpha, t)$ of the random variable

$$
\frac{1}{n} \sum_{k \leq n t} V\left(\xi+\frac{S_{k}}{\sqrt{n}}\right)
$$

then $\sigma_{\xi}(\alpha, t)$ also satisfies (2 and 3), but now the condition $\psi^{\prime}(+0)-\psi^{\prime}(-0)=-2$ is replaced by $\psi^{\prime}(\xi+)-\psi^{\prime}(\xi-)=-2$. Therefore, repeating steps (4 to 7 ), we get

$$
\int_{0}^{\infty} e^{-\alpha} d_{\alpha} \sigma_{\xi}(\alpha, t)=\sum_{j} e^{-\lambda_{j} t} \psi_{j}(\xi) \int_{-\infty}^{\infty} \psi_{j}(x) d x .
$$

Thus

$$
\frac{\psi_{1}(\xi)}{\psi_{1}(0)}=\lim _{t \rightarrow \infty} \frac{\int_{0}^{\infty} e^{-\alpha} d_{\alpha} \sigma_{\xi}(\alpha, t)}{\int_{0}^{\infty} e^{-\alpha} d_{\alpha} \sigma(\alpha, t)},
$$

so that we are also able to calculate the principal eigenfunction.

The extension of the preceding method to multidimensional Schrödinger equations is immediate. It is in these cases that the method will probably prove to be most useful since, unlike the standard variational procedures, the extension to several dimensions seems to cause comparatively little difficulty. For illustrative purposes we will consider Schrödinger's equation in three dimensions. Here we must consider three independent sequences

$$
\begin{aligned}
& X_{1}, X_{2}, X_{3}, \ldots, \\
& Y_{1}, Y_{2}, Y_{3}, \ldots, \\
& Z_{1}, Z_{2}, Z_{3}, \ldots,
\end{aligned}
$$

of independent identically distributed random variables each having mean 0 and standard deviation 1. Let $S_{x k}=X_{1}+X_{2}+\cdots+X_{k}$, and $S_{y k}$, $S_{z k}$ have the obvious meanings. We consider the limiting distributions $\sigma(\alpha, t)$ and $\sigma(\alpha, \xi, \eta, \zeta, t)$ of the random variables

$$
\frac{1}{n} \sum_{k \leq n t} V\left(\frac{S_{x k}}{\sqrt{n}}, \frac{S_{y k}}{\sqrt{n}}, \frac{S_{z k}}{\sqrt{n}}\right)
$$

and

$$
\frac{1}{n} \sum_{k \leq n t} V\left(\xi+\frac{S_{x k}}{\sqrt{n}}, \eta+\frac{S_{y k}}{\sqrt{n}}, \zeta+\frac{S_{z k}}{\sqrt{n}}\right),
$$

respectively.

In exactly the same way as in the one dimensional case, we arrive at

$$
\lambda_{1} \sim \frac{1}{t_{2}-t_{1}} \log \frac{\int_{0}^{\infty} e^{-\alpha} d_{\alpha} \sigma\left(\alpha, t_{1}\right)}{\int_{0}^{\infty} e^{-\alpha} d_{\alpha} \sigma\left(\alpha, t_{2}\right)},
$$

and

$$
\frac{\psi_{1}(\xi, \eta, \zeta)}{\psi_{1}(0,0,0)}=\lim _{t \rightarrow \infty} \frac{\int_{0}^{\infty} e^{-\alpha} d_{\alpha} \sigma(\alpha, \xi, \eta, \zeta, t)}{\int_{0}^{\infty} e^{-\alpha} d_{\alpha} \sigma(\alpha, t)} .
$$

So far, the theory was carried out under the assumption that the potential function $V$ was nonnegative. In most cases of physical interest this is not so. For the hydrogen atom, for instance

$$
V(x, y, z)=-\frac{\text { const. }}{\sqrt{x^{2}+y^{2}+z^{2}}} \cdot
$$

However, the modification is easy, although we have not as yet cleared up all the points of mathematical rigor.

The formula for the lowest eigenvalue now becomes

$$
\lambda_{1} \sim \frac{1}{t_{2}-t_{1}} \log \frac{\int_{-\infty}^{0} e^{-\alpha} d_{\alpha} \sigma\left(\alpha, t_{1}\right)}{\int_{-\infty}^{0} e^{-\alpha} d_{\alpha} \sigma\left(\alpha, t_{2}\right)},
$$

and a corresponding modification needs to be made in the formula for the principal eigenfunction. We have not yet tested numerically any case with a negative potential function, but we hope to be able to report on this in the near future.

\section{Numerical Examples and Discussion}

The Monte Carlo procedure used here consists in the calculation of the distribution function $\sigma(\alpha, t)$ by a sampling process; the principal eigenvalue is then calculated from (10). For the purposes of numerical illustration we consider two examples, $V(x)=x^{2}$ and $V(x)=|x|$. In both of 
these cases the eigenvalues are known, and hence we have a check on the accuracy of our procedure. In order to calculate $\sigma(\alpha, t)$, say when $V(x)=x^{2}$, we see from (1) that we must consider the limiting distribution as $n \rightarrow \infty$ of

$$
\frac{1}{n^{2}} \sum_{k \leq n t} S_{k}^{2}
$$

This means from our point of view that we must (a) choose a distribution for the $X$ 's, (b) choose a sufficiently large $n$, (c) select an appropriate $t$, (4) calculate for $n t X$ 's the normalized sum (14), (5) repeat (4) many times, so that the empirical distribution may be obtained from these many samples.

Although, under the conditions mentioned previously, the distribution function $\sigma(\alpha, t)$ is independent of the distribution of the $X$ 's, the actual numerical calculation of $\sigma(\alpha, t)$ is expedited by choosing the distribution of the $X$ 's to be the Bernoulli distribution, i. e.,

$$
P(X=1)=P(X=-1)=\frac{1}{2} .
$$

The sequence of random variables $X_{1}, X_{2}, X_{3}$, ... is then a sequence of +1 's and -1 's, such as might be obtained in coin tossing. This is conveniently and rapidly achieved on a calculating machine by considering sequences of random digits, counting even digits +1 and odd digits -1 .

The value of $n$ to be used must be large enough so that the empirical distribution function calculated is close to the theoretical limiting distribution function $\sigma(\alpha, t)$. From (10) we see that the two values of $t, t_{1}$, and $t_{2}$, to be used in the calculation of $\lambda$ must be large enough so that the exponential terms neglected are sufficiently small. However, since the sample size is $n t$, the desire to make both $n$ and $t$ large must be tempered by practical considerations. The number of samples to be used must be large enough so that the empirical distribution adequately represents $\sigma(\alpha, t)$. Before discussing these points in more detail we consider an actual numerical computation. The following data for $V(x)=x^{2}$ and $V(x)=|x|$ were calculated from a certain set of random digits ${ }^{6}$ on the IBM Electronic Calculating Punch, Type 604. For both $x^{2}$ and $|x| n$ was selected to be 400 ,

6 This 'set of random digits was prepared by the RAND Corporation, Santa_Monica, Calif. $t_{1}=5, t_{2}=3.75$, and 100 samples were used. In table 1 is tabulated

$$
\begin{aligned}
& A_{1}=\frac{1}{8000} \sum_{k=1}^{1500}\left|S_{k}\right|, \quad A_{2}=\frac{1}{8000} \sum_{k=1}^{2000}\left|S_{k}\right|, \\
& B_{1}=\frac{1}{160000} \sum_{k=1}^{1500} S_{k}^{2}, \quad B^{2}=\frac{1}{160000} \sum_{k=1}^{2000} S_{k}^{2} .
\end{aligned}
$$

Since each RAND random number card contains 50 random digits, and 2,000 digits are needed to form one sample, a set of 4,000 RAND cards was sufficient for this experiment. It takes $20 \mathrm{~min}$ utes on the IBM 604 to calculate $A_{1}$ and $A_{2}$ for one sample (similarly for $B_{1}$ and $B_{2}$ ), so that it

\begin{tabular}{|c|c|c|c|c|}
\hline & $A_{1}$ & $A_{2}$ & $B_{1}$ & $B_{2}^{\prime}$ \\
\hline 1 & 1. 332 & 2. 101 & 0.738 & 1. 290 \\
\hline 2 & 3.808 & 5. 707 & 5. 295 & 8.502 \\
\hline 3 & 2. 795 & 5. 545 & 3. 460 & 9.610 \\
\hline 4 & 8. 723 & 13.917 & 30.722 & 52. 816 \\
\hline 5 & 4. 169 & 4. 766 & 6.123 & 6.602 \\
\hline 6 & 1.195 & 2. 136 & .598 & 1.453 \\
\hline 7 & 6.674 & 12.112 & 15. 258 & 39.058 \\
\hline 8 & 4. 103 & 5. 242 & 5.826 & 7.004 \\
\hline 9 & 5. 751 & 8.840 & 10.440 & 18.143 \\
\hline 10 & 4. 250 & 4. 981 & 6.069 & 6.679 \\
\hline 11 & 2. 909 & 5. 643 & 3.844 & 10.044 \\
\hline 12 & 2.834 & 3.416 & 3. 602 & 4. 097 \\
\hline 13 & 1.888 & 2. 194 & 1. 595 & 1. 700 \\
\hline 14 & 2.022 & 2. 337 & 1. 510 & 1. 638 \\
\hline 15 & 1.680 & 3. 908 & 1. 184 & 5. 289 \\
\hline 16 & 7. 700 & 12. 712 & 24. 769 & 44. 936 \\
\hline 17 & 3. 228 & 4. 973 & 3.563 & 6.145 \\
\hline 18 & 1.844 & 2.654 & 1. 523 & 2. 183 \\
\hline 19 & 2.376 & 5. 275 & 2. 017 & 8.854 \\
\hline 20 & 4. 533 & 5.640 & 6.380 & 7. 616 \\
\hline 21 & 4. 209 & 7. 543 & 7. 735 & 16. 703 \\
\hline 22 & 3.847 & 5.590 & 6.666 & 9. 204 \\
\hline 23 & 5. 206 & 6. 314 & 9.148 & 10.600 \\
\hline 24 & 1. 962 & 2. 698 & 1. 859 & 2. 923 \\
\hline 25 & 3.523 & 4. 807 & 5.084 & 6. 482 \\
\hline 26 & 3. 605 & 5. 397 & 5.014 & 7. 680 \\
\hline 27 & 9.168 & 13. 692 & 27.973 & 44. 461 \\
\hline 28 & 5.625 & 7.898 & 11. 528 & 16.010 \\
\hline 29 & 3. 215 & 4. 152 & 3.362 & 4. 170 \\
\hline 30 & 3. 293 & 3.972 & 4. 136 & 4. 677 \\
\hline 31 & 2. 710 & 3.683 & 3. 006 & 4. 025 \\
\hline 32 & 3. 638 & 7. 386 & 6.114 & 17.413 \\
\hline 33 & 7. 928 & 11. 567 & 25.084 & 36.001 \\
\hline 34 & 3.475 & 3.846 & 4. 396 & 4. 550 \\
\hline 35 & 1.958 & 2. 594 & 1. 457 & 1. 948 \\
\hline 36 & 5. 356 & 7. 216 & 8.888 & 11. 781 \\
\hline 37 & 3. 297 & 6.395 & 3.800 & 11.555 \\
\hline 38 & 1.883 & 2. 682 & 1.387 & 1.995 \\
\hline 39 & 4. 849 & 10.020 & 8. 396 & 30.233 \\
\hline 40 & 2. 282 & 3.067 & 2. 258 & 2. 882 \\
\hline
\end{tabular}
takes approximately 35 hours to secure the following data.

TABLE 1. Experimental data for lowest eigenvalue

Journal of Research 
TABLE 1. Experimental data for lowest eigenvalue-Con.

\begin{tabular}{|c|c|c|c|c|}
\hline & $A_{1}$ & $A_{2}$ & $B_{1}$ & $B_{2}$ \\
\hline 41 & 4. 253 & 6.957 & 6. 744 & 13.502 \\
\hline 42 & 7.559 & 8.834 & 17.143 & 18. 702 \\
\hline 43 & 1.122 & 2. 587 & .500 & 2. 634 \\
\hline 44 & 1.433 & 3. 602 & .869 & 4.855 \\
\hline 45 & 2.984 & 3.477 & 2.851 & 3.161 \\
\hline 46 & 1. 677 & 3.158 & 1.025 & 3.341 \\
\hline 47 & 5.145 & 9.697 & 11.941 & 28.826 \\
\hline 48 & 4.982 & 9.140 & 9. 201 & 23.092 \\
\hline 49 & 1.675 & 2. 152 & 1. 129 & 1. 472 \\
\hline 50 & 1. 580 & 3.375 & 1. 147 & 3.807 \\
\hline 51 & 2. 606 & 3. 621 & 2. 254 & 3. 247 \\
\hline 52 & 4.303 & 5.883 & 7.987 & 10.104 \\
\hline 53 & 7.553 & 12.092 & 19. 632 & 36.427 \\
\hline 54 & 3.138 & 5. 703 & 3.968 & 9.325 \\
\hline 55 & 3.343 & 4. 327 & 4. 264 & 5.380 \\
\hline 56 & 1.423 & 2.315 & 0.770 & 1. 533 \\
\hline 57 & 7.996 & 11. 458 & 21.405 & 31.585 \\
\hline 58 & 1.916 & 2.744 & 1. 253 & 1. 959 \\
\hline 59 & 1.490 & 2.161 & 0.789 & 1. 404 \\
\hline 60 & 3.536 & 5.382 & 4. 125 & 7. 461 \\
\hline 61 & 6. 278 & 11.060 & 16.187 & 34. 779 \\
\hline 62 & 1.174 & 1. 709 & 0.573 & 0.919 \\
\hline 63 & 5. 272 & 9.919 & 10.463 & 27.825 \\
\hline 64 & 3.576 & 5.277 & 6.021 & 8. 707 \\
\hline 65 & 2. 686 & 5.081 & 3.577 & 8.307 \\
\hline 66 & 6.166 & 8.785 & 14.056 & 19.627 \\
\hline 67 & 4. 412 & 5. 548 & 6.095 & 7.182 \\
\hline 68 & 1. 732 & 4. 664 & 1. 170 & 8. 720 \\
\hline 69 & 2. 730 & 4. 970 & 2.440 & 6. 639 \\
\hline 70 & 1.331 & 2. 772 & 0.765 & 2. 642 \\
\hline 71 & 3.668 & 5. 318 & 5.730 & 8.001 \\
\hline 72 & 2.948 & 3.801 & 3. 248 & 4. 104 \\
\hline 73 & 5. 279 & 8. 931 & 12.455 & 23.513 \\
\hline 74 & 11. 284 & 15. 784 & 41. 684 & 58.525 \\
\hline 75 & 2.183 & 2. 787 & 2.086 & 2. 455 \\
\hline 76 & 4. 298 & 7.427 & 9.139 & 17. 157 \\
\hline 77 & 1.412 & 2.893 & 0.894 & 3.490 \\
\hline 78 & 2.002 & 2.567 & 1. 602 & 2. 024 \\
\hline 79 & 2.619 & 3.780 & 2. 981 & 4. 205 \\
\hline 80 & 2. 695 & 4. 616 & 2.974 & 6.077 \\
\hline 81 & 4. 706 & 6.672 & 8.330 & 11. 487 \\
\hline 82 & 5.517 & 7. 981 & 9.846 & 14. 805 \\
\hline 83 & 1.755 & 2.099 & 1.066 & 1. 207 \\
\hline 84 & 2.084 & 2.876 & 1. 735 & 2. 504 \\
\hline 85 & 1.574 & 2. 743 & 0.993 & 2. 256 \\
\hline 86 & 5.818 & 10.444 & 13.322 & 30.522 \\
\hline 87 & 4. 387 & 7.844 & 9.037 & 18.695 \\
\hline 88 & 3.666 & 5. 244 & 4. 416 & 6.541 \\
\hline 89 & 7.111 & 13.311 & 19. 834 & 50.822 \\
\hline 90 & 6.280 & 8.548 & 13. 403 & 17.587 \\
\hline 91 & 4.872 & 5.836 & 9. 230 & 10.023 \\
\hline 92 & 3.035 & 3.323 & 2. 865 & 2. 973 \\
\hline 93 & 3.608 & 4. 697 & 4. 256 & 5. 357 \\
\hline 94 & 3.977 & 4.964 & 5. 215 & 6. 242 \\
\hline 95 & 3.614 & 7.867 & 5. 757 & 20.434 \\
\hline 96 & 9.193 & 11.183 & 27. 789 & 31.075 \\
\hline 97 & 4.108 & 5. 282 & 5. 918 & 7. 374 \\
\hline 98 & 4. 355 & 5. 744 & 7. 774 & 10. 223 \\
\hline 99 & 1.898 & 2.661 & 1. 507 & 2. 013 \\
\hline 100 & 9.683 & 17.154 & 35.803 & 80.543 \\
\hline
\end{tabular}

In order to calculate $\lambda_{1}$ from(10) we need the values of $\int_{0}^{\infty} e^{-\alpha} d_{\alpha} \sigma\left(\alpha, t_{1}\right)$ and $\int_{0}^{\infty} e^{-\alpha} d_{\alpha} \sigma\left(\alpha, t_{2}\right)$. Both of these integrals were calculated numerically from the data in table 1 by adding the exponentials of the entries in appropriate columns. For the case $V(x)=|x|$, the values of the integrals were obtained from $1 / 100 \sum_{i=1}^{100} e^{-A_{1 j}}$ and $1 / 100 \sum_{j=1}^{100} e^{-A_{2 i}}$ and for $V(x)$ $=x^{2}$ from $1 / 100 \sum_{j=1}^{100} e^{-B_{1 i}}$ and $1 / 100 \sum_{j=1}^{100} e^{-B_{2 i}}$. In the case $V(x)=|x|$ the true lowest eigenvalue to two places is 0.81 , and in the case $V(x)=x^{2}$ it is $\sqrt{2} / 2=$ 0.71 .

\begin{tabular}{|c|r|r|r|}
\hline & $\begin{array}{r}\text { First 50 } \\
\text { samples }\end{array}$ & $\begin{array}{c}\text { Second 50 } \\
\text { samples }\end{array}$ & $\begin{array}{r}\text { All 100 } \\
\text { samples }\end{array}$ \\
\hline & 0.83 & 0.79 & 0.81 \\
$|x|$ & .80 & .69 & .75 \\
$x^{2}$ & .69 & \\
\hline
\end{tabular}

An interesting feature of the data is that column $B_{2}$, for example, may be thought of as arising from $n=2000, t=1 ; n=1000, t=2$, etc., as well as from $n=400, t=5$. The larger we take $n$, the closer will be the empirical distribution to $\sigma(\alpha, t)$. The value of $\int_{0}^{\infty} e^{-\alpha} d_{\alpha} \sigma(\alpha, t)$ in the case $t=1$, and $V(x)$ $=x^{2}$ can be calculated exactly and, to three places, is 0.678. Calculating this integral from column $B_{2}$ with $n=2000, t=1$, we get 0.685 .

Instead of using Bernoulli distributed variables, one might use other distributions. One definite advantage of Bernoulli distributed variables is that the computation utilizes only the crudest properties of the random digits, i. e., whether they are even or odd. One possible advantage for certain other distributions is that $n$ might not have to be taken so large. In particular this should be true if we use Gaussian distributed variables. RAND Gaussian deviates were used in constructing table 2. Here we chose $t_{1}=3.75, t_{2}=5$ as before, but now $n=100$. This means samples of size 500 instead of 2000 and therefore a total machine computation time of 9 hours for 100 samples.

Envisaging the possibility of calculating the second eigenvalue, we considered the quantities

$$
10^{-4} \sum_{k=1}^{375}\left(S_{k}+5\right)^{2} \text { and } 10^{-4} \sum_{k=1}^{500}\left(S_{k}+5\right)^{2},
$$


TABLE 2. Experimental data for second eigenvalue

\begin{tabular}{|c|c|c|c|c|c|}
\hline & $C_{1}$ & $C_{2}$ & & $C_{1}$ & $C_{2}$ \\
\hline 1 & 3.963 & 13. 997 & 61 & 49. 929 & 84.307 \\
\hline 2 & 2. $348^{*}$ & 2. 721 & 62 & 3.095 & 6. 220 \\
\hline 3 & $1.889^{*}$ & 2. 251 & 63 & 2. $585^{*}$ & $3.867^{*}$ \\
\hline 4 & $3.817^{*}$ & 4. $372^{*}$ & 64 & 1. $318^{*}$ & 1. $906^{*}$ \\
\hline 5 & 2. 395 & 2. $802^{*}$ & 65 & 2. $241^{*}$ & $9.102^{*}$ \\
\hline 6 & 12. 467 & 27. 683 & 66 & 9.737 & 12.185 \\
\hline 7 & 1. $068^{*}$ & 1. $115^{*}$ & 67 & 9. $738^{*}$ & 11. $347^{*}$ \\
\hline 8 & $0.578^{*}$ & $0.967^{*}$ & 68 & 4. 604 & 5. 589 \\
\hline 9 & 1. $504^{*}$ & 1. 760 & 69 & 20.364 & 29. 707 \\
\hline 10 & 4. 249 & 9. 787 & 70 & 1. $097^{*}$ & 1. $895^{*}$ \\
\hline 11 & 1. $751^{*}$ & 3. $316^{*}$ & 71 & 5.891 & 13.391 \\
\hline 12 & 9.922 & 13.975 & 72 & 9. $019^{*}$ & $12.815^{*}$ \\
\hline 13 & 5. 680 & 6.649 & 73 & 1. 843 & 6. 280 \\
\hline 14 & 3.348 & 4. 684 & 74 & 7. 961 & 11.431 \\
\hline 15 & 13.431 & 24. 769 & 75 & 1. 109 & 1. 926 \\
\hline 16 & 1. $473^{*}$ & 2. $615^{*}$ & 76 & 2. $417^{*}$ & 3.037 \\
\hline 17 & 45.262 & 74. 304 & 77 & 2. $402^{*}$ & 2. $797^{*}$ \\
\hline 18 & 1. $157^{*}$ & 3.109 & 78 & $2.043^{*}$ & 4. $214^{*}$ \\
\hline 19 & $0.906^{*}$ & $3.644^{*}$ & 79 & $0.582^{*}$ & 0.752 \\
\hline 20 & 4. 601 & 12. 641 & 80 & 2. 429 & 4.381 \\
\hline 21 & 8. $356^{*}$ & 13. $084^{*}$ & 81 & 8.584 & 13. 054 \\
\hline 22 & $0.308^{*}$ & 1. $874^{*}$ & 82 & 2. $793^{*}$ & 4. $659^{*}$ \\
\hline 23 & 2. $111^{*}$ & 4. $428^{*}$ & 83 & 1.557 & 5.023 \\
\hline 24 & 2. $035^{*}$ & 2. 726 & 84 & 1. $006^{*}$ & 1. $677^{*}$ \\
\hline 25 & 1. $625^{*}$ & 3.898 & 85 & $5.327^{*}$ & 15. $531^{*}$ \\
\hline 26 & 1. $879^{*}$ & 5. 713 & 86 & 2. 058 & 7.307 \\
\hline 27 & 1. 333 & 8. 961 & 87 & 12.481 & $12.803^{*}$ \\
\hline 28 & 1. 769 & $2.319^{*}$ & 88 & $0.925^{*}$ & 2.403 \\
\hline 29 & 1. $906^{*}$ & 2. $417^{*}$ & 89 & 15.519 & 19.879 \\
\hline 30 & 7.522 & 7. $979^{*}$ & 90 & 1. $456^{*}$ & 4. $009^{*}$ \\
\hline 31 & 14. 934 & 15. $493^{*}$ & 91 & 5. 244 & 10.047 \\
\hline 32 & 4. $282^{*}$ & 13. $747^{*}$ & 92 & 2. 497 & 2. 931 \\
\hline 33 & 1. $356^{*}$ & 2. 718 & 93 & 16. 700 & 17.382 \\
\hline 34 & 4. 448 & 8. 329 & 94 & 4. $471^{*}$ & $8.156^{*}$ \\
\hline 35 & 1. $306^{*}$ & 2. $112^{*}$ & 95 & 2. $139^{*}$ & 2. $580^{*}$ \\
\hline 36 & 5. $465^{*}$ & $12.396^{*}$ & 96 & 1. $196^{*}$ & 2. $273^{*}$ \\
\hline 37 & 2. 918 & 9.006 & 97 & 23.014 & 28.420 \\
\hline 38 & 35.821 & 56. 626 & 98 & 6. $959^{*}$ & 7. $022^{*}$ \\
\hline 39 & $9.814^{*}$ & 11. $707^{*}$ & 99 & $3.374^{*}$ & 8. $651^{*}$ \\
\hline 40 & 1.811 & 4. 628 & 100 & 1. $600^{*}$ & -2. $250^{*}$ \\
\hline 41 & 3.374 & 6.460 & 101 & 4. $626^{*}$ & 7. $794^{*}$ \\
\hline 42 & 1. 633 & 6.578 & 102 & 5. $195^{*}$ & 10. $215^{*}$ \\
\hline 43 & 6. 976 & 8. 705 & 103 & $6.837^{*}$ & $12.160^{*}$ \\
\hline 44 & 7. 595 & 13. 096 & 104 & 1. 913 & 4. $412^{*}$ \\
\hline 45 & 2.336 & 2. 862 & 105 & 2. $276^{*}$ & 2. 770 \\
\hline 46 & 1. $931^{*}$ & 2. $579^{*}$ & 106 & 1. $935^{*}$ & 3. $983^{*}$ \\
\hline 47 & 4. $507^{*}$ & 8. $294^{*}$ & 107 & 3.560 & 5.808 \\
\hline 48 & $8.581^{*}$ & 11. $301^{*}$ & 108 & $2.857^{*}$ & 7. $384^{*}$ \\
\hline 49 & 4. $698^{*}$ & 8. $220^{*}$ & 109 & 3. $399^{*}$ & 4. $484^{*}$ \\
\hline 50 & 10.154 & 16.884 & & & \\
\hline & & & 110 & 6. $917^{*}$ & $27.107^{*}$ \\
\hline 51 & 8.828 & 15.387 & 111 & 38.661 & 56.479 \\
\hline 52 & $0.997^{*}$ & 1. $604^{*}$ & 112 & 1.891 & $2.000^{*}$ \\
\hline 53 & 8.582 & 12.838 & 113 & 8.506 & 9. $646^{*}$ \\
\hline 54 & 2. $730^{*}$ & 3. $249^{*}$ & 114 & 4. 209 & 10.461 \\
\hline 55 & 10. 471 & 13.140 & 115 & 33.194 & 44. 247 \\
\hline 56 & 1. $456^{*}$ & $1.845^{*}$ & 116 & $0.869^{*}$ & 1. 289 \\
\hline 57 & 8. $230^{*}$ & 11. $924^{*}$ & 117 & 3. 946 & 15. 204 \\
\hline 58 & $5.515^{*}$ & 8. $146^{*}$ & 118 & 1. $680^{*}$ & 2. $995^{*}$ \\
\hline 59 & 2. $202^{*}$ & 3. $182^{*}$ & 119 & $8.178^{*}$ & 9.056 \\
\hline 60 & 2. $129^{*}$ & 9. $650^{*}$ & 120 & 27.621 & 51.650 \\
\hline
\end{tabular}

TABLE 2. Experimental data for second eigenvalue-Con.

\begin{tabular}{|c|c|c|c|c|c|}
\hline & $C_{1}$ & $C_{2}$ & & $C_{1}$ & $C_{2}$ \\
\hline 121 & $8.444^{*}$ & 15. $836^{*}$ & 161 & 3. 546 & 4. $040^{*}$ \\
\hline 122 & 13. 254 & 16. 467 & 162 & 1. $238^{*}$ & 2. $304^{*}$ \\
\hline 123 & 1. $755^{*}$ & 5. $246^{*}$ & 163 & $0.975^{*}$ & 1.283 \\
\hline 124 & 36.902 & 70.134 & 164 & 1. 980 & 3. $659^{*}$ \\
\hline 125 & $7.567^{*}$ & 18. $553^{*}$ & 165 & 14. $620^{*}$ & $17.565^{*}$ \\
\hline 126 & 14. 725 & 38.551 & 166 & $2.378^{*}$ & 2.865 \\
\hline 127 & $0.983^{*}$ & 2. 572 & 167 & 2. 279 & 6. 279 \\
\hline 128 & 24.529 & 31.660 & 168 & 10.846 & 13. 754 \\
\hline 129 & 2. $042^{*}$ & 2. $385^{*}$ & 169 & 1. $341^{*}$ & 3. $673^{*}$ \\
\hline 130 & 3. $587^{*}$ & $9.858^{*}$ & 170 & 1. $861^{*}$ & 2. $729^{*}$ \\
\hline 131 & 1. $335^{*}$ & 1. 755 & 171 & $28.588^{*}$ & $53.358^{*}$ \\
\hline 132 & 2. $142^{*}$ & $3.801^{*}$ & 172 & 2.943 & $3.087^{*}$ \\
\hline 133 & 16. 361 & 27. 759 & 173 & 1. 694 & 2.568 \\
\hline 134 & 8.193 & 9. 301 & 174 & 1. 729 & $2.516^{*}$ \\
\hline 135 & 2. $719^{*}$ & 5. $141^{*}$ & 175 & $0.844^{*}$ & 1. $449^{*}$ \\
\hline 136 & 4. $767^{*}$ & $12.737^{*}$ & 176 & $3.441^{*}$ & 5. $380^{*}$ \\
\hline 137 & 42.230 & 108. 038 & 177 & 0.712 & $0.838^{*}$ \\
\hline 138 & 41. 236 & 78.423 & 178 & 1. $222^{*}$ & $3.469^{*}$ \\
\hline 139 & 8. 243 & 9. $911^{*}$ & 179 & $20.852^{*}$ & $42.263^{*}$ \\
\hline 140 & 31.522 & 36. 393 & 180 & $7.575^{*}$ & $16.552^{*}$ \\
\hline 141 & 2. 600 & 2. $881^{*}$ & 181 & $2.042^{*}$ & 3.058 \\
\hline 142 & 1.932 & 2. $055^{*}$ & 182 & 6. 797 & 7. $343^{*}$ \\
\hline 143 & 8.562 & 10.813 & 183 & 1. 536 & 4. 499 \\
\hline 144 & $1.421^{*}$ & 3. $652^{*}$ & 184 & 1. 235 & $1.472^{*}$ \\
\hline 145 & 2. $669^{*}$ & 3. $889^{*}$ & 185 & $3.541^{*}$ & 4. 272 \\
\hline 146 & $1.125^{*}$ & 2. 071 & 186 & 5. $394^{*}$ & $6.149^{*}$ \\
\hline 147 & $3.439^{*}$ & 5. $824^{*}$ & 187 & 18.589 & 28.787 \\
\hline 148 & 1. $269^{*}$ & 5. 651 & 188 & 8.372 & 12.628 \\
\hline 149 & 7.949 & 14. 252 & 189 & $13.470^{*}$ & $23.775^{*}$ \\
\hline 150 & 2. $808^{*}$ & 4. $434^{*}$ & 190 & 1. $196^{*}$ & $2.851^{*}$ \\
\hline 151 & 6.413 & 12.573 & 191 & 3. 507 & 7. 712 \\
\hline 152 & 5. $936^{*}$ & $10.432^{*}$ & 192 & 21.489 & 43.372 \\
\hline 153 & 9.788 & 18. 093 & 193 & 1. $038^{*}$ & 1. $109^{*}$ \\
\hline 154 & 1. 251 & 2. 707 & 194 & $3.591^{*}$ & 3.864 \\
\hline 155 & 34. 799 & 63. 494 & 195 & 2. $295^{*}$ & 2. $529^{*}$ \\
\hline 156 & $0.841^{*}$ & 1. $742^{*}$ & 196 & 1. $044^{*}$ & 2.320 \\
\hline 157 & 1. $461^{*}$ & 1. $615^{*}$ & 197 & 4. $865^{*}$ & $6.759^{*}$ \\
\hline 158 & 7.220 & 21.125 & 198 & 1.184 & 11.852 \\
\hline 159 & 1. $275^{*}$ & 2. $359^{*}$ & 199 & 1. $228^{*}$ & 1. $969^{*}$ \\
\hline 160 & 9.355 & 19. 565 & 200 & $9.346^{*}$ & $22.204^{*}$ \\
\hline
\end{tabular}

which correspond to taking $\xi=0.5$ in (11). This should not make any difference in the calculation of the lowest eigenvalue, and consequently table 2 can be utilized in the same way as table 1 . It should however be borne in mind that columns $C_{1}$ and $C_{2}$ of table 2 represent experimental values of the quantities (18) with the $S_{k}$ 's being sums of Gaussian deviates. Asterisks on the entries of table 2 indicate that $S_{375}$ or $S_{500}$ was negative. Although this information is unnecessary for the purpose of calculations of the lowest eigenvalue, it is used in the calculation of the next eigenvalue. How this can be done is explained briefly in section IV. 
Using the data in table 2 and again (10) we computed the following approximations to $\lambda_{1}$ (actual value $=0.71$ )

\begin{tabular}{|c|c|c|c|c|c|}
\hline & $\begin{array}{c}\text { First 50 } \\
\text { samples }\end{array}$ & $\begin{array}{c}\text { Second 50 } \\
\text { samples }\end{array}$ & $\begin{array}{c}\text { Third 50 } \\
\text { samples }\end{array}$ & $\begin{array}{c}\text { Fourth 50 } \\
\text { samples }\end{array}$ & $\begin{array}{c}\text { All 200 } \\
\text { samples }\end{array}$ \\
\cline { 1 - 3 }$\lambda_{1}$ & 0.82 & 0.72 & 0.83 & 0.64 & 0.74 \\
\hline
\end{tabular}

For both $V(x)=|x|$ and $V(x)=x^{2}$, all the eigenvalues are known so that in the two illustrative examples above it was easy to choose appropriate values for $t_{1}$ and $t_{2}$. The proper value for $n$ and the appropriate number of samples was determined experimentally. In general when all eigenvalues are unknown the following "rule of thumb" procedure seems to be feasible. Having first made a guess at an appropriate $n$ and $t$, and a certain number of samples, compute $\lambda_{1}$. Repeat the computation now for the same $n$, the same number of samples and successively larger $t$ 's until the calculated values of $\lambda_{1}$ become stable to the desired number of places. If they do not become stable, the number of samples must be increased. Keeping two values of $t$, for which the calculated $\lambda$ 's had the stable value, increase $n$ and see if the calculated value of $\lambda$ changes. If not, $n$ is sufficiently large. If it does change, increase $n$ until a new stability appears. This stable value is then the appropriate approximation to $\lambda_{1}$. The mere fact that stability is observed means the number of samples is sufficiently large.

\section{The Second Eigenvalue}

If the principal eigenfunction is even, then it is possible to extend the theory of section II in such a way that the calculation of the second eigenvalue becomes feasible. Without going into any details we just state the pertinent result: Let
$E_{t}^{+}(\xi)=\lim _{n \rightarrow \infty} E\left\{e^{\left\{\frac{1}{n} \sum_{k \leq n t} V\left(\xi+\frac{S_{k}}{\sqrt{n}}\right)\right.} \mid S_{n t}>0\right\}$,

and

$E_{t}^{-}(\xi)=\lim _{n \rightarrow \infty} E\left\{e^{-\frac{1}{n} \sum_{k \leq n t} V\left(\xi+\frac{S_{k}}{\sqrt{n}}\right)} \mid S_{n t}<0\right\}$,

the mathematical expectations on the right hand sides being conditional expectations under the conditions $S_{n t}>0$ and $S_{n t}<0$, respectively.

We then have

$\frac{1}{2}\left\{E_{t}^{+}(\xi)-E_{t}^{-}(\xi)\right\}=\sum_{j=2}^{\infty} e^{-\lambda_{j} t} \psi_{j}(\xi) \int_{-\infty}^{\infty} \rho(x) \psi_{j}(x) d x$,

where

$$
\rho(x)=\left\{\begin{array}{l}
+1, x>0 \\
-1, x<0
\end{array}\right.
$$

If $t_{1}$ and $t_{2}$ are sufficiently large, we get

$$
\lambda_{2} \sim-\frac{1}{t_{2}-t_{1}} \log \frac{E_{t_{2}}^{+}(\xi)-E_{t_{2}}^{-}(\xi)}{E_{t_{1}}^{+}(\xi)-E_{t_{1}}^{-}(\xi)} .
$$

From the discussion of section III it should be clear how one applies (19) when the data of table 2 are available. One must see to it that $\xi$ is so chosen that $\psi_{2}(\xi) \neq \equiv$, otherwise a higher eigenvalue may have been calculated. From the data of table 2 we obtain

$$
\lambda_{2} \sim 1.1
$$

whereas the exact value is $3 / 2 \sqrt{2}=2.12$. The poor agreement could have been expected in view of low accuracy in the calculation of $\lambda_{1}$.

In conclusion, we thank E. C. Yowell, of the National Bureau of Standards, for wiring the boards and for excellent supervision of all the punched card work.

Los Angeles, September 12, 1949. 